\title{
A NOVEL TECHNIQUE FOR MULTIPLE FAULTS AND THEIR LOCATIONS DETECTION AND START ELECTRODE SELECTION IN MICROFLUIDIC DIGITAL BIOCHIP
}

\author{
MUKTA MAJUMDER \\ Department of Computer Science and Engineering \\ Birla Institute of Technology \\ Mesra, Ranchi, Jharkhand, India \\ mukta_jgec_it_4@yahoo.co.in \\ NILANJANA DAS \\ Maynaguri CCC, WBSEDCL, Jalpaiguri \\ West Bengal, India \\ nilanjanadas_2010@yahoo.com \\ SUJAN KUMAR SAHA \\ Department of Computer Science and Engineering \\ Birla Institute of Technology \\ Mesra, Ranchi, Jharkhand, India \\ sujan.kr.saha@gmail.com
}

Received 21 June 2013

Accepted 22 July 2013

Published 22 August 2013

\begin{abstract}
A device, that is used for biomedical operation or safety-critical applications like point-of-care health assessment, massive parallel DNA analysis, automated drug discovery, air-quality monitoring and food-safety testing, must have the attributes like reliability, dependability and correctness. As the biochips are used for these purposes; therefore, these devices must be fault free all the time. Naturally before using these chips, they must be well tested. We are proposing a novel technique that can detect multiple faults, locate the fault positions within the biochip, as well as calculate the traversal time if the biochip is fault free. The proposed technique also highlights a new idea how to select the appropriate base node or pseudo source (start electrode). The main idea of the proposed technique is to form multiple loops with the neighboring electrode arrays and then test each loop by traversing test droplet to check whether there is any fault. If a fault is detected then the proposed technique also locates it by backtracking the test droplet. In case, no fault is detected, the biochip is fault free then the proposed technique also calculates the time to
\end{abstract}

This is an Open Access article published by World Scientific Publishing Company. It is distributed under the terms of the Creative Commons Attribution 3.0 (CC-BY) License. Further distribution of this work is permitted, provided the original work is properly cited. 
traverse the chip. The result suggests that the proposed technique is efficient and shows significant improvement to calculate fault-free biochip traversal time over existing method.

Keywords: Biochip; lab-on-chip; medical microsystems; biochemical droplet; biomedical operation.

\section{Introduction}

For biochemical analysis or bio-assay operations digital microfluidic biochips (DMFB) have become very popular in recent times. ${ }^{1-3}$ This biochip is also called lab-on-a-chip or bio-MEMS, as it replaces highly repetitive laboratory tasks by replacing traditional bulky lab equipments with composite microsystem..$^{1-4}$ It offers the advantages of design flexibility, higher sensitivity, smaller size and lower cost. ${ }^{1-5}$ Naturally in last few years' biochips or bioMEMS catch a fair deal of attraction from the researchers.

In earlier generation, microfluidic biochip was based on the manipulation of continuous fluid flow carried out by using micropumps, microvalves and microchannels ${ }^{2-4}$; an alternate approach is to manipulate liquids as discrete droplet. ${ }^{4-7}$ The dropletbased chip is referred as "Digital Microfluidic Biochip". ${ }^{1}$ The latter one is advantageous over the continuous flow Systems. ${ }^{3,4,6}$

Correctness and Dependability are the important attributes for DMFB that are used as Medical Microsystems, for biomedical operation or safetycritical applications like point-of-care health assessment, massive parallel DNA analysis, automated drug discovery, air-quality monitoring, foodsafety testing, etc. Therefore, these devices must be tested after manufacturing and during bio-assay operations. Since these biochips are being targeted for a highly competitive and low-cost market segment, test and diagnosis methods must be inexpensive, quick and effective. In this paper, we are proposing an efficient cost-effective technique that can detect multiple faults and locate these faults within the biochip; and it can also calculate the time to traverse a fault-free biochip using multiple droplets; beside these the proposed technique also highlights a new idea how to select the appropriate base node or pseudo source (start electrode) for traversing the biochip.

Remaining paper is organized as Sec. 2 discusses the preliminaries of DMFB and graph theoretic formulation. Section 3 discusses the related prior works. Section 4 represents the proposed technique. Section 5 presents a brief simulation result of the proposed technique. And conclusions are drawn at Sec. 6.

\section{Preliminaries of DMFB and Graph Theoretic Formulation}

The digital microfluidic devices consist of a twodimensional (2D) array of electrodes with one or more sources and sinks on the boundary and a droplet of some biochemical sample. ${ }^{2,8}$ These biochips are based on the manipulation of micro-nano liter droplets on a 2D electrode array using the principle of electrowetting. ${ }^{1,5,9,10}$ This biochemical droplet can be moved by applying a control voltage to an electrode just adjacent to the droplet and, at the same time, deactivating the electrode just under the droplet. This electronic phenomenon of wettability control creates interfacial tension gradient that moves the droplets toward the charged electrode. Using the electrowetting phenomenon, droplets can be moved to any location on a $2 \mathrm{D}$ electrode array plate. It can achieve a speed upto $20 \mathrm{~cm} / \mathrm{s}$ under a control voltage between 0 and $90 \mathrm{~V} .^{5,10,11}$ By varying the patterns and directions of control voltage many fluidic handling operations can be done such as droplet merging, splitting, mixing, etc. Basic structure of such a biochip is described below.

\subsection{Structure of the biochip}

The basic cell of a microarray includes a pair of electrodes that acts as two parallel plates. The bottom plate contains an array of individually controllable electrodes; the top plate is coated with a continuous ground electrode. Hydrophobic dielectric insulator is added to the plates to decrease wettability of the surfaces and to increase capacitance between a droplet and a plate. A biochemical droplet rests on a hydrophobic surface over an electrode, and is sandwiched between two parallel 


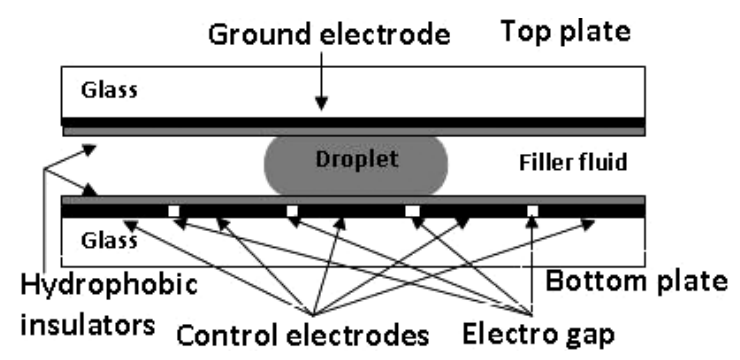

Fig. 1. Structure of a DMFB.
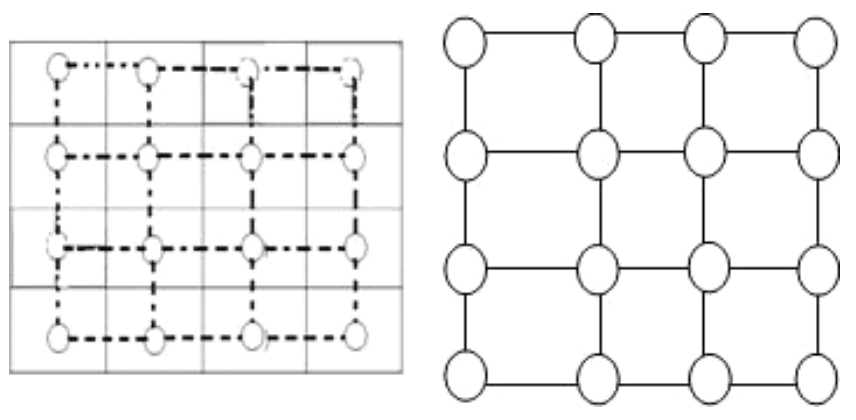

Fig. 2. $4 \times 4$ Microfluidic array represented as $4 \times 4$ matrix or Graph.

glass plates. $^{1,8-11}$ The basic cell structure of a digital microfluidic-based biochip is shown in Fig. 1.

\subsection{Graph theoretic formulation}

Now we can represent a DMFB in terms of an $m \times$ $n$ array (2D matrix) where $C_{i j}$ denotes the cell at $(i, j)$ position, and $i=0$ to $m-1 ; j=0$ to $n-1$.

At any moment a droplet can be at any cell in the microarray. Hence the $m \times n$ microfluidic biochip can be represented in terms of a graph $G_{m \times n}$ (or $2 \mathrm{D}$ matrix) where $m$ is the number of rows and $n$ is number of columns. Figure 2 shows the graphical representation of a $4 \times 4$ microfluidic biochip.

\section{Related Prior Work}

Testing of microfluidic device or MEMS is a new topic in the area of researches, as the microfluidic technology itself is a new one. A welldescribed previous work on the testing of microfluidic devices can be found in Ref. 12. Methods on fault modeling and fault simulation for continuousflow microfluidic biochips have been presented by Kerkhoff et al. in Refs. 13 and 14. For classifying defects and test application procedures for digital biochip have been discussed by $\mathrm{Su}$ et al. in Ref. 15; faults in biochip have been categorized as catastrophic and parametric. For detecting catastrophic faults in digital microfluidic arrays, some effective techniques have been found in Refs. 2 and 10. A concurrent testing methodology for detection of catastrophic faults in digital microfluidic systems has been presented and the problems of test planning and resource optimization have been discussed by Chakraborty et al. in Ref. 2. In Ref. 16, Davids et al. has discussed a technique for faults detection and their diagnosis in biochip. The technique has three basic steps. In the first step, the outer loop of the microfluidic array has been tested using one single droplet. In the second step, all the columns of the microarray have been tested using multiple droplets in parallel. And in the third step the rows have been tested using the same technique used for column testing. Fault detections in microfluidic biochips with multiple droplets in parallel have also being discussed in Refs. 8, 17, 18 and 19. In Refs. 8, 17 and 19 the proposed techniques, first select some start electrodes (pseudo sources) or base nodes, then the traversal of the microarray is done by moving the droplets from these pseudo sources or base nodes. So the appropriate selections of base nodes or pseudo sources are very much important. Though these techniques have used pseudo source or base node the issues of exiting fault in pseudo source or base node have not been taken care. In Ref. 20, a technique has been proposed to use a Hamiltonian path to detect catastrophic faults in microfluidic arrays. One problem with this approach is that although finding Hamiltonian paths in grid structures is well known, but checking the existence of Hamiltonian path in a given graph is NP-complete problem. So it would be very expensive to determine such paths in the microfluidic array. An alternative method for testing digital microfluidic biochip based on Euler paths is found in Ref. 21 . This method maps a DMFB to an undirected graph and an Euler path is determined for testing the biochip. A technique of multiple faults detection and identification of their locations has been proposed by Davids et al. in their paper Ref. 6. In Ref. $22 \mathrm{Xu}$ and Chakrabarty has he presented different fundamental operations of biochip like droplet dispensing, droplet transportation, mixing, splitting and capacitive sensing. 


\section{Proposed Technique}

In this paper, we are proposing a novel technique for identifying the appropriate start electrode (base node) for traversing the microarray. The proposed technique can detect and locate multiple faults within a microfluidic array. It can also calculate the time which is required to traverse a fault-free biochip. The technique first checks whether any fault is there within the biochip, and if a fault is detected, it also locates the position of the fault within the chip and if there is no fault then it will calculate the traversal time of the microarray.

\subsection{Placing droplet and loop formation}

First of all the test droplet is dispensed from the source reservoirs and placed on the start electrodes at the microarray boundary as row and column wise, such a way that there must be one electrode gap between every two droplets placed on start electrodes, to avoid merging or mixing of them as shown in Figs. 3 and 4. Selection strategy of start electrode is very much important; we select those electrodes which are fault-free. To select a fault-free electrode, a capacitive detection circuit and a droplet reservoir are connected with a test electrode. Capacitive sensing is a technique by which we can identify the presence of a droplet at a test electrode $^{23,24}$ A droplet is dispensed from source reservoir and placed on the test electrode. Then the neighboring edges are checked by traversing the droplet to the next electrode and backtrack it as shown in Fig. 3. The capacitive detection circuit is used to verify the presence of test droplet. In Fig. 3(a) electrode $(0,0)$ is fault free but $(0,2)$ and $(2,0)$ are faulty and in Fig. 3(b) $(0,3),(3,1)$ and $(3,3)$ all the

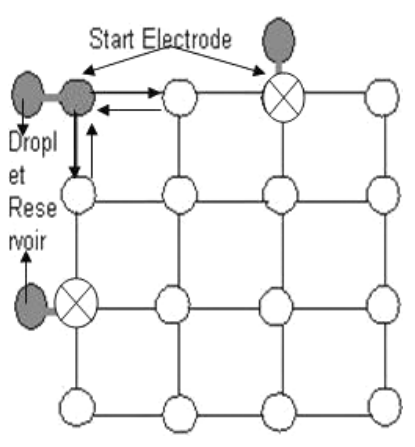

(a)

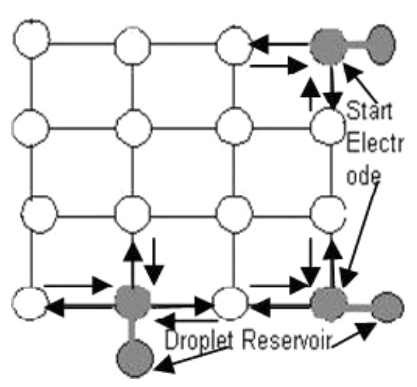

(b)
Fig. 3. Selection of start electrode.

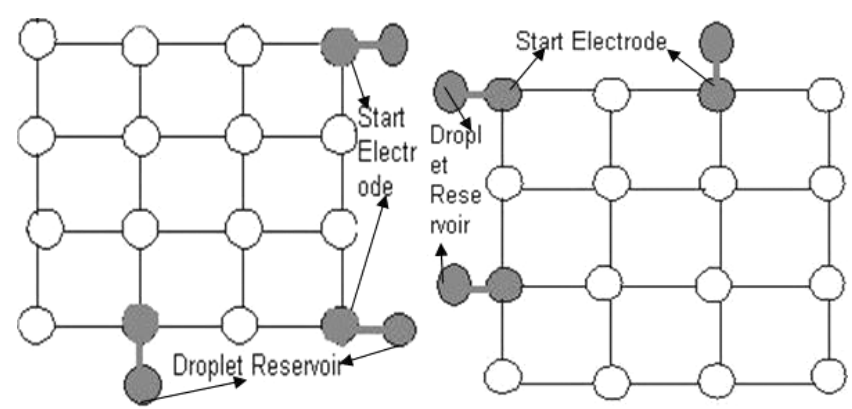

Fig. 4. Droplets placed on start electrode dispensed from source.

three electrodes are fault free. So in this case the start electrodes selection is shown as in Fig. 3(b).

If the test electrode and at least one neighboring edge are fault free, the electrode may be selected as start electrode depending upon the suitable placement of droplet and grouping of neighboring electrodes for loops formation. Every start electrode is connected with a droplet reservoir for easy placement of droplet and a capacitive detection circuit that can detect the presence of a test droplet at the start electrode.

We are considering columns and rows as the target region of these droplets. Suppose there are $m$ number of rows and $n$ number of columns. Now all the rows and columns are numbered as 0 to $(m-1)$ and 0 to $(n-1)$. If there are even number of rows and columns then each neighboring two rows and two columns are grouped together to form loops, depending upon the suitable placement of droplets at fault-free start electrodes, as shown in Fig. 5(b).

If there are odd number of rows and columns then except one row and one column, all are grouped together to form loops as discussed before, depending upon the suitable placement of droplets

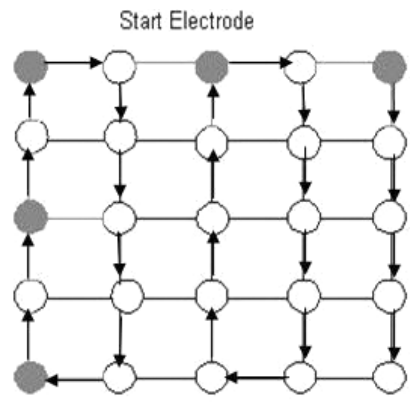

(a)

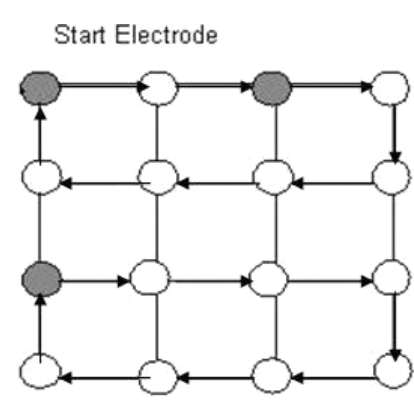

(b)
Fig. 5. Loop formation and traversing droplet in clockwise direction. 
at fault-free start electrodes, as shown in Fig. 5(a). After the loops are formed, the microarray is ready for testing. The testing methodology is discussed below.

\subsection{Testing methodology}

Now each loop is tested by traversing the droplet from source to sink which are eventually same for a particular loop (except odd electrode). Here the start electrode acts as source and sink. Multiple faults detection is achieved by traversing each and every loop twice by applying control voltage to the electrode, in clockwise and anticlockwise direction. We are assuming that one edge movement is equivalent to one time unit. A test droplet starting from the source is routed through a loop by traversing all the vertices and their respective edges and guided to the sink to complete a loop traversal. If the biochemical droplet does not reach the sink which is eventually the source (except odd electrode), after a certain period of time, say $T$ (suppose droplet takes $T$ time to traverse a loop), we can consider that a fault exists in the loop. The droplet will stop there; further forward movement of the droplet is not possible in this case; otherwise, it will be guided back to the sink as shown in Fig. 6(a) by the bold arrowed line.

Presence of fault in a microarray is indicated by a cross mark in Fig. 6(b). The sink electrode, which is also the source electrode in this case, (except odd electrode) is connected to a capacitive detection circuit that can identify the presence of the test droplet.

Once a fault is detected in the loop, it is necessary to locate this fault. An attempt is made to locate this fault by applying a control voltage to the electrode in opposite direction that is the test droplet is backtrack in anticlockwise direction as shown in Fig. 6(b) by the dotted line. We know that a droplet takes one unit time for one edge movement, so we can conclude that if a droplet takes 4 unit of time for backtracking from the fault position to source electrode, then the fault is identified at 4 unit distance from the source electrode as depicted in Fig. 6(b).

Suppose time $t_{1}$ is taken for the backtracking operation. If the droplet takes $T$ time to traverse a same size fault-free loop then $\left(T-t_{1}\right)$ is the time the droplet will take to traverse the other half of the loop. Now if a fault is detected in forward traversal process in one half of the loop, then there may be another fault that exists in other half of the loop. This implies the necessity of checking the other half of the loop of the microarray. The other half of the loop is traversed by moving the droplet in anticlockwise direction as shown by bold arrowed line in Fig. 7(a). Now the previous fault position is known and we can calculate the time $t_{2}\left(T-t_{1}\right)$ that the droplet will take to reach the previous fault location. After the time out, the droplet is backtrack in clockwise direction as shown by dotted line in Fig. 7(b). During backtracking if the droplet takes $t_{3}$ time and it is less than $t_{2}$, we can conclude that there is another fault in the other half of the loop.

If there are odd number of rows and columns in a microarray then the row and column electrode which are not grouped with any other electrodes are traversed only in clockwise direction and backtracking is done in anticlockwise direction, in case if any fault is there the scenario is shown in Fig. 8(b). The other half of the odd electrode is checked in the

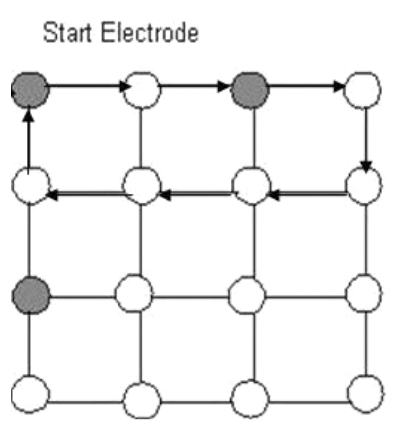

(a)

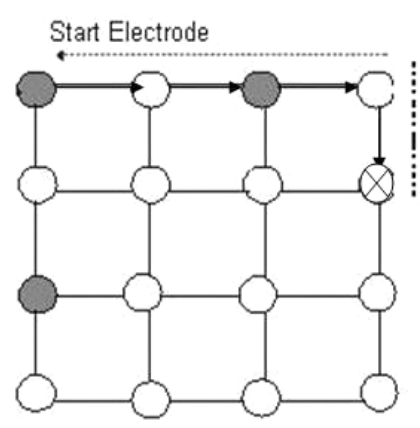

(b)

Fig. 6. Clockwise movement of droplet in microarray with a fault-free and a faulty loop and anticlockwise backtrack.

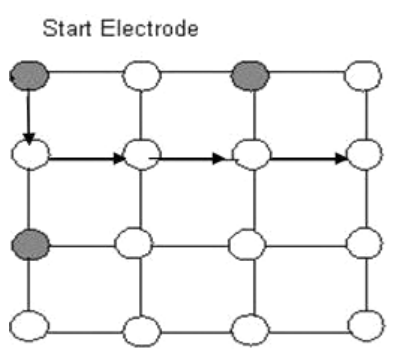

(a)

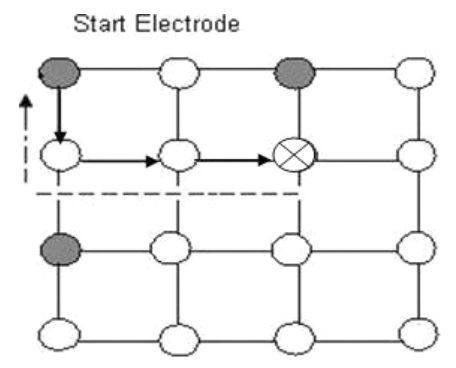

(b)
Fig. 7. Anticlockwise movement of droplet with a fault-free and a faulty loop and clockwise backtrack. 


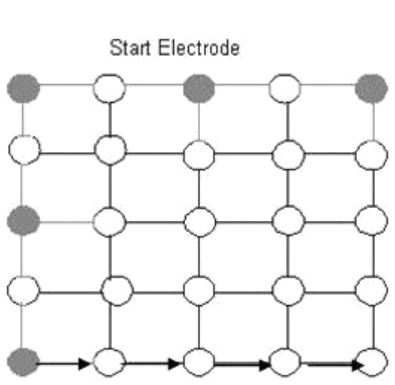

(a)

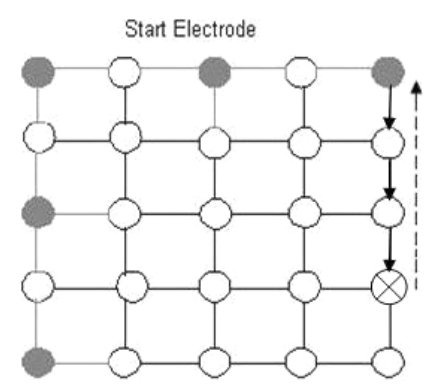

(b)
Fig. 8. Last electrode traversal in case of odd number of rows and columns in the microarray.

similar way. This traversal technique is applied to both row and column electrodes to detect multiple faults and their locations.

The procedure of the proposed technique which detects and locates multiple faults within the biochip is discussed below.

\section{Procedure for Traversal Technique Begin}

1. Dispense droplets from reservoir and placed on start electrode by spacing one electrode gap. $\downarrow^{*}$ To avoid merging of droplet $* \backslash$

2 . If rows and columns are even numbered

Form $L$ number of loops with two neighboring electrodes.

\section{Else}

Form a self loop with the odd electrode and $L-1$ loops with rest of other neighboring electrodes.

3. While all $L$ loops are not traversed Repeat steps 4 to 5 .

4. Move the droplets to traverse a loop in clockwise direction from source to sink and calculate time $T$.

5. If the droplet stops somewhere and does not reach the sink within time out period $T .1^{*}$ Then a fault is there $* \backslash$

Backtrack the droplet in anti clock wise direction and calculate time $t_{1} \cdot l^{*}$ To locate the fault position $* \backslash$

If a fault is found in the loop

Move the droplets from same source to traverse the same loop in anti clock wise direction and calculate time $t_{2}$ $\left(T-t_{1}\right) . \perp^{*}$ To check if any more fault is there in the same loop $* \backslash$

After time out backtrack the droplet in clock wise direction and calculate time $t_{3} .{ }^{*}$ To check if another fault is there ${ }^{*} \backslash$

If backtracking time $t_{3}$ is less than $t_{2}\left(T-t_{1}\right)$ then the droplet was stopped at $t_{3}$ unit distance before previous fault position. ${ }^{*}$ To locate another fault $* \backslash$

End of step 3 loop.

End.

\section{Result}

The proposed technique can detect at most two faults within a single loop. Row-wise and column-wise loops are traversed in sequentially to avoid merging or mixing of the test droplets. But a certain type of loops, for example row loops or column loops are traversed at the same time or in parallel. We know that one edge movement of droplet is equivalent to one time unit. So the total time to traverse a fault-free microarray is the sum total of row-wise loops traversal time plus column-wise loops traversal time.

The simulation is done in Turbo $\mathrm{C}$ environment by considering the structure of microarray chip as 2D matrices. Table 1 shows some experimental calculated times to traverse a vast number of microarrays starting from $4 \times 4$.

\subsection{Analysis}

We have considered the row- and column-wise loops traversal in sequential order for avoiding the issue of merging or mixing of droplets and calculated the total time required to traverse the microarray, as sum of these two. But if we consider the condition

Table 1. Time taken to traverse a fault-free biochip.

\begin{tabular}{ccccc}
\hline $\begin{array}{l}\text { Serial } \\
\text { no. }\end{array}$ & $\begin{array}{c}\text { Size of } \\
\text { biochip }\end{array}$ & $\begin{array}{c}\text { Time for } \\
\text { row traversal }\end{array}$ & $\begin{array}{c}\text { Time for } \\
\text { column traversal }\end{array}$ & $\begin{array}{c}\text { Total } \\
\text { time }\end{array}$ \\
\hline 1 & $4 \times 4$ & 8 & 8 & 16 \\
2 & $4 \times 5$ & 10 & 8 & 18 \\
3 & $5 \times 5$ & 10 & 10 & 20 \\
4 & $5 \times 6$ & 12 & 10 & 22 \\
5 & $6 \times 6$ & 12 & 12 & 24 \\
6 & $6 \times 7$ & 14 & 12 & 26 \\
7 & $7 \times 7$ & 14 & 14 & 28 \\
8 & $7 \times 8$ & 16 & 14 & 30 \\
9 & $8 \times 8$ & 16 & 16 & 32 \\
10 & $8 \times 9$ & 18 & 16 & 34 \\
11 & $9 \times 9$ & 18 & 18 & 36 \\
12 & $10 \times 10$ & 20 & 20 & 40 \\
\hline
\end{tabular}


Table 2. Comparison with two existing techniques.

\begin{tabular}{lcccc}
\hline Serial no. & Size & Existing & Proposed & Improvement \\
\hline 1 & $4 \times 4$ & 48 & 23 & 52.08 \\
2 & $4 \times 5$ & 66 & 25 & 62.12 \\
3 & $5 \times 5$ & 88 & 27 & 69.31 \\
4 & $5 \times 6$ & 112 & 29 & 74.10 \\
5 & $6 \times 6$ & 140 & 31 & 77.85 \\
6 & $6 \times 7$ & 170 & 33 & 80.58 \\
7 & $7 \times 7$ & 204 & 35 & 82.84 \\
8 & $7 \times 8$ & 240 & 37 & 84.58 \\
9 & $8 \times 8$ & 280 & 39 & 86.07 \\
10 & $8 \times 9$ & 322 & 41 & 87.26 \\
\hline
\end{tabular}

where merging and mixing of droplets are not important issues, then we can use parallel traversal for row and column loops like in Refs. 17, 18 and 22; then the time required for testing the microarray will be much lesser. In Refs. 8, 17 and 19 the authors have formulated the solutions strategies by considering only one droplet source reservoir; test droplets are dispensed from this reservoir and placed on pseudo sources or base nodes. But it is also important to consider the fact that to place a test droplet on pseudo source or base node; we have to move the droplet from reservoir to pseudo source or base node following a path. Now before testing the microarray we cannot predict that this path is fault free and the pseudo source or base node itself is fault free. Naturally, we have selected individual droplet reservoirs for each and every pseudo source or base node (start electrode) and also identify appropriate fault-free pseudo source or base node.

We have presented a comparative study of the proposed technique with a technique ${ }^{6}$ that can also detect multiple faults within a biochip in the following Table 2, considering the sequential scanning of the microarray. Time for selecting the start electrode (pseudo source) has been also added to the total traversing time. According to Fig. 3(b) a start electrode (base node) on microarray boundary can have maximum of three neighboring nodes. Now to select a pseudo source (base node) following steps are involved:

1. Dispense droplet from reservoir and place it on start electrode.

2. Neighboring edges are checked by traversing droplet to the next electrode and backtrack it.

Now we are assuming that dispensing of droplet and placing it on start electrode takes one time unit. We have previously considered that one edge movement of droplet takes one unit of time. So according to Fig. 3(b) selecting a start electrode (pseudo source) which has maximum of three neighboring nodes will take 7 unit of time.

\section{Conclusions}

An advanced, novel and efficient technique for detecting and locating multiple faults in digital microfluidic-based biochips has been presented in this paper. It has also been shown that the proposed technique is able to calculate the traversal time for a fault-free biochip. The proposed technique also discussed how to identify appropriate fault-free start electrode (pseudo source) or base node before using them for traversing the microarray. It also takes care about merging or mixing of test droplets during traversal. As the biochips are used as medical microsystem, for biomedical operation and safety-critical analysis like health assessment, massive parallel DNA analysis, automated drug discovery, air-quality monitoring, food-safety testing, etc., the fault detection and identification of those fault locations in microfluidic biochip are very much important.

\section{References}

1. F. Su, K. Chakrabarty, R. B. Fair, "Microfluidicsbased biochips: Technology issues, implementation platforms, and design-automation challenges," IEEE Trans. Comput.-Aided Des. Integrated Circ. Syst. 25(2), 211-223 (2005).

2. F. S. S. Ozev, K. Chakraborty, "Concurrent testing of droplet-based microfluidic systems for multiplexed biomedical assays," ITC International Test Conf. pp. 883-892 (2004).

3. F. Su et al., "Architectural-level synthesis of digital microfluidic based biochips," Proc. IEEE Int. Conf. on CAD, pp. 223-228 (2004).

4. S. Fei et al., "Defect-oriented testing and diagnosis of digital microfluidics-based biochips," Proc. IEEE Int. Test Conf. pp. 1-10 (2005).

5. M. Pollack, A. Shenderov, R. B. Fair, "Electro wetting-based actuation of droplets for integrated micro-fluidics," Lab Chip 2, 96-101 (2002).

6. D. Davids et al., "Multiple fault diagnosis in digital microfluidic biochips," ACM J. Emerg. Technol. Comput. Syst. 2(4), 1-15 (2006).

7. V. Srinivasan, V. Pamula, R. Fair, "An integrated digital micro-fluidic lab-on-a-chip for clinical diagnostics on human physiological fluids," Lab Chip 310-315 (2004). 
8. T. $\mathrm{Xu}$ and K. Chakraborty, "Parallel scan-like testing and fault diagnosis techniques for digital microfluidic biochips," IEEE Trans. Biomed. Circuits Syst. 1(2), 148-158 (2007).

9. R. B. Fair et al., "Electro wetting-based on-chip sample processing for integrated micro-fluidics," Proc. IEDM, pp. 32.5.1-32.5.4 (2003).

10. F. Su et al., "Testing and diagnosis of realistic defects in digital microfluidic biochip," J. Electron. Test. Theory Appl. 23, 219-233 (2007).

11. F. Su, S. Ozev, K. Chakraborty, "Test planning and test resource optimization for droplet-based microfluidic systems," J. Electron. Test. Theory Appl. 22, 199-210 (2006).

12. H. G. Kerkhoff, "Testing of microelectronic-bio-fluidic systems," IEEE Design Test Comput. 24, 72-82 (2007).

13. H. G. Kerkhoff, H. P. A. Hendriks, "Fault modeling and fault simulation in mixed micro-fluidic microelectronic systems," J. Electron. Testing Theory Appl. 17, 427-437 (2001).

14. H. G. Kerkhoff, M. Acar, "Testable design and testing of micro-electro-fluidic arrays," Proc. IEEE VLSI Test Symp. pp. 403-409 (2003).

15. F. Su, S. Ozev, K. Chakraborty, "Testing of dropletbased microfluidic systems," Proc. IEEE Int. Test Conf. pp. 1192-1200 (2003).

16. D. Davids, B. Joshi, A. Mukherjee, A. Ravindran, "A fault detection and diagnosis technique for digital micro-fluidic biochips," IMS3TW '08 Proc. IEEE 14th International Mixed-Signals, Sensors, and Systems Test Workshop, pp. 1-4 (2008).
17. S. Saha, A. Chakraborty, S. Roy, "An efficient single fault detection technique for microfluidic based biochip," Int. Conf. Advances in Computer Engineering, pp. 10-14 (2010).

18. M. Majumder, K. Hansda, S. Roy, "A novel singlefault detection technique of digital micro-fluidic biochip," Int. J. Comput. Sci. Appl. 1, 92-95 (2011).

19. M. Majumder, S. Ray, S. Roy, "A multi droplets detection technique for single-fault in digital microfluidic biochip," CIIT 2011, CCIS Vol. 250, pp. 337342, Springer-Verlag Berlin Heidelberg (2011).

20. T. H. Schulte, R. L. Bardell, B. H. Weigl, "Microfluidic technologies in clinical diagnostics," Clin. Chim. Acta 321, 1-10 (2002).

21. A. Itai et al., "Hamilton paths in grid graphs," SIAM J. Comput. 11, 676-686 (1982).

22. T. Xu, K. Chakrabarty, "Functional testing of digital microfluidic biochips," Test Conf. ITC. IEEE Int. pp. 1-10 (2007).

23. H. Kai, B.-N. Hsu, A. Madison, K. Chakrabarty, R. Fair, "Fault detection, real-time error recovery, and experimental demonstration for digital microfluidic biochips," Proc. Conf. Design, Automation and Test in Europe, pp. 559-564, EDA Consortium (2013).

24. B. Hadwen, G. R. Broder, D. Morganti, A. Jacobs, C. Brown, J. R. Hector, Y. Kubota, H. Morgan, "Programmable large area digital microfluidic array with integrated droplet sensing for bioassays," Lab Chip 12(18), 3305-3313 (2012). 